\title{
Elective Nodal Irradiation for Non-small Cell Lung Cancer Complicated With Chronic Obstructive Pulmonary Disease Affects Immunotherapy After Definitive Chemoradiotherapy
}

\author{
MASAHIRO MORIMOTO ${ }^{1}$, KAZUMI NISHINO $^{2}$, KENTARO WADA $^{1}$, FUMIO IMAMURA $^{2}$, KOJI KONISHI ${ }^{1}$, \\ HANAKO KUHARA ${ }^{2}$, MOTOHIRO TAMIYA ${ }^{2}$, TAKAKO INOUE ${ }^{2}$, KEI KUNIMASA ${ }^{2}$, MADOKA KIMURA ${ }^{2}$, \\ TAKERO HIRATA ${ }^{1,3}$, NAOYUKI KANAYAMA ${ }^{1}$, MASAYASU TORATANI ${ }^{1}$, HAYATO KAWACHI $^{2}$, \\ KIKA OHIRA ${ }^{2}$, ERINA NAKANISHI ${ }^{1,3}$, SHINGO OHIRA ${ }^{1}$, TOMOHIRO SAGAWA ${ }^{1}$, \\ MASAYOSHI MIYAZAKI ${ }^{1}$, TAKASHI MATSUNAGA ${ }^{4}$, TORU KUMAGAI ${ }^{2}$ and TERUKI TESHIMA ${ }^{1}$ \\ Department of ${ }^{1}$ Radiation Oncology, ${ }^{2}$ Thoracic Oncology, ${ }^{4}$ Medical Informatics, \\ Osaka International Cancer Institute, Osaka, Japan; \\ ${ }^{3}$ Department of Radiation Oncology, Osaka University Graduate School of Medicine, Osaka, Japan
}

\begin{abstract}
Background/Aim: The aim of this retrospective study was to detect the frequency, reasons, and significant factors for not receiving immunotherapy after chemoradiotherapy in non-small cell lung cancer (NSCLC) patients. Patients and Methods: Thirty-four patients with NSCLC received definitive chemoradiotherapy. The endpoint of this study was receiving durvalumab within 45 days after chemoradiotherapy for NSCLC. Results: Twenty-five of 34 (73\%) patients received immunotherapy within 45 days after chemoradiotherapy. The reasons for not receiving immunotherapy were radiation pneumonitis (50\%), radiation esophagitis (10\%), and four other reasons (40\%). Univariate analysis showed that significant factors for not receiving immunotherapy were elective nodal irradiation $(E N I)+$ and chronic obstructive pulmonary disease $(C O P D)+$. The rate of immunotherapy was 100\% (17/17 cases) in the COPDand ENI- group, and 16\% (1/6 cases) in the COPD+ and ENI+ group. Conclusion: ENI for NSCLC complicated with COPD decreased the rate of immunotherapy after definitive chemoradiotherapy.
\end{abstract}

This article is freely accessible online.

Correspondence to: Masahiro Morimoto, Department of Radiation Oncology, Osaka International Cancer Institute, 3-1-69 Otemae, Chuo-ku, Osaka 541-8567, Japan. Tel: +81 669451181, Fax: +81 69451900, e-mail: morimoto-knk@umin.ac.jp

Key Words: Volumetric modulated arc therapy (VMAT), intensity modulated radiation therapy (IMRT), involved field radiotherapy irradiation (IFR-IFI), twice-daily thoracic radiotherapy (BID-TRT), accelerated hyperfractionated thoracic radiotherapy (AHTRT), antiprogrammed cell death ligand-1 immune checkpoint inhibitor (ICIs).
The anti-programmed cell death ligand-1 immune checkpoint inhibitor, durvalumab, is a standard treatment for locally advanced non-small cell lung cancer (NSCLC) after chemoradiotherapy $(1,2)$. However, not all NSCLC patients can receive immunotherapy after chemoradiotherapy for various reasons. In clinical practice, reasons include adverse events from chemoradiotherapy, progressive disease, or other factors.

The characteristics of patients who cannot receive immunotherapy and the significant factors that are correlated with not receiving immunotherapy are not clear. The aim of this retrospective study was to detect the frequency, reasons, and significant factors for not receiving immunotherapy after chemoradiotherapy in NSCLC patients.

\section{Patients and Methods}

A total of 34 patients with NSCLC received definitive chemoradiotherapy between May 2018 and January 2019. Institutional review board approval was obtained for this study (number 19020). The patient characteristics and treatment factors are listed in Table I. The diagnosis of NSCLC was confirmed by histologic findings. The patients underwent the following pretherapeutic procedures: a physical examination and chest radiography; fiberoptic bronchoscopy and endobronchial ultrasonography as needed; complete blood cell count and biochemical tests including tumor markers; electrocardiography; computed tomography (CT) imaging of the chest and abdomen; positron emission tomography/CT. A hematologic status, which included a white blood cell count $>3,500 / \mathrm{mm}^{3}$, platelet count $>100,000 / \mathrm{mm}^{3}$, and hemoglobin level $>10 \mathrm{~g} / \mathrm{dl}$, was generally required. When febrile neutropenia or grade 3 non-hematologic adverse events excluding nausea, vomiting, appetite loss, and elevated creatinine or liver enzymes developed, radiotherapy was generally interrupted. Adverse events were evaluated using Common Terminology Criteria for Adverse Events, Version 4.0. 
Table I. Patient characteristics and treatment factors.

\begin{tabular}{|c|c|c|c|}
\hline & $\mathrm{N}$ & & $\mathrm{N}$ \\
\hline Number of patients & 34 & Hemoglobin (g/dl) & \\
\hline Age (years) & & Median & 13.4 \\
\hline Median & 73 & Range & $9.6-15.7$ \\
\hline Range & $30-87$ & White blood cell count $/ \mathrm{mm}^{3}$ & \\
\hline Gender & & Median & 6,520 \\
\hline Male & $25(73 \%)$ & Range & $3,890-10,790$ \\
\hline Female & $9(26 \%)$ & Neutrophil cell count $/ \mathrm{mm}^{3}$ & \\
\hline Performance status & & Median & 4,140 \\
\hline 0 & $27(79 \%)$ & Range & $2,060-9,080$ \\
\hline 1 & $7(20 \%)$ & Platelet cell count $/ \mathrm{mm}^{3}$ & \\
\hline Location of primary site & & Median & 229,500 \\
\hline Upper lobe & $22(64 \%)$ & Range & $150,000-451,000$ \\
\hline Middle lobe & $1(2 \%)$ & C-reactive protein $(\mathrm{mg} / \mathrm{dl})$ & \\
\hline Lower lobe & $11(32 \%)$ & Median & 0.16 \\
\hline $\mathrm{T}$ classification & & Range & $0.02-9.45$ \\
\hline 0 & $1(2 \%)$ & Creatinine clearance $(\mathrm{ml} / \mathrm{min})$ & \\
\hline $1 \mathrm{a}$ & $1(2 \%)$ & Median & 73 \\
\hline $1 \mathrm{~b}$ & $6(17 \%)$ & Range & $31-128$ \\
\hline $1 \mathrm{c}$ & $1(2 \%)$ & Carcinoembryonic antigen (ng/ml) & \\
\hline $2 \mathrm{a}$ & $5(14 \%)$ & Median & 4.7 \\
\hline $2 b$ & $6(17 \%)$ & Range & $1-322$ \\
\hline 3 & $6(17 \%)$ & Cytokeratin 19 fragment $(\mathrm{ng} / \mathrm{ml})$ & \\
\hline 4 & $8(23 \%)$ & Median & 3.4 \\
\hline $\mathrm{N}$ classification & & Range & $0.9-25.1$ \\
\hline 0 & $5(14 \%)$ & Sialylated carbohydrate antigen KL-6 (U/ml) & \\
\hline 1 & $8(23 \%)$ & Median & 259 \\
\hline 2 & $17(50 \%)$ & Range & $103-669$ \\
\hline 3 & $4(11 \%)$ & Number of patients with epidermal & $2(5 \%)$ \\
\hline Stage & & growth factor receptor mutation & \\
\hline IA2 & $2(5 \%)$ & Number of patients with anaplastic & $2(5 \%)$ \\
\hline IB & $1(2 \%)$ & lymphoma kinase fusion gene & \\
\hline IIA & $1(2 \%)$ & Number of patients with c-ros & $3(8 \%)$ \\
\hline IIB & $5(14 \%)$ & oncogene 1 fusion gene & \\
\hline IIIA & $14(41 \%)$ & Programmed cell death 1 ligand & \\
\hline IIIB & $9(26 \%)$ & 1 expression level $(\%)$ & \\
\hline IIIC & $2(5 \%)$ & Median & 50 \\
\hline Histology & & Range & $0-95$ \\
\hline Adenocarcinoma & $18(52 \%)$ & Vital capacity (1) & \\
\hline Squamous cell carcinoma & $15(44 \%)$ & Median & 3.14 \\
\hline Pleomorphic carcinoma & $1(2 \%)$ & Range & $1.68-5.42$ \\
\hline Positron emission tomography standardized & & \%vital capacity & \\
\hline maximum uptake value & & Median & 100 \\
\hline Median & 12 & Range & $60.7-134$ \\
\hline Range & $2-37.5$ & Forced expiratory volume in $1 \mathrm{sec}(\mathrm{l})$ & \\
\hline Brinkman index & & Median & 1.92 \\
\hline Median & 945 & Range & $1.17-4.2$ \\
\hline Range & $0-4200$ & Forced expiratory volume in $1 \mathrm{sec} \%$ & \\
\hline Number of patients with asthma & $2(5 \%)$ & Median & 75 \\
\hline Number of patients with diabetes mellitus & $6(17 \%)$ & Range & $42-91$ \\
\hline $\begin{array}{l}\text { Number of patients complicated } \\
\text { with interstitial pneumonia }\end{array}$ & $2(5 \%)$ & $\begin{array}{l}\% \text { predicted forced expiratory volume in } 1 \mathrm{sec} \\
\text { Median }\end{array}$ & 75 \\
\hline Number of patients complicated with & $18(52 \%)$ & Range & $52-164$ \\
\hline emphysematous change on computed tomography & & Number of patients complicated with & $11(32 \%)$ \\
\hline Number of patients with post-operative recurrence & $7(20 \%)$ & chronic obstructive pulmonary disease & \\
\hline Number of patients with a history of drinking & $23(67 \%)$ & Number of patients complicated with & $5(14 \%)$ \\
\hline Body mass index $\left(\mathrm{kg} / \mathrm{m}^{2}\right)$ & & mild chronic obstructive pulmonary & \\
\hline Median & 21.5 & disease $(\%$ predicted forced expiratory & \\
\hline Range & $16.3-30.1$ & volume in 1 second $\geq 80 \%$ ) & \\
\hline
\end{tabular}




\begin{tabular}{|c|c|}
\hline & $\mathrm{N}$ \\
\hline $\begin{array}{l}\text { Number of patients complicated with } \\
\text { intermediate chronic obstructive } \\
\text { pulmonary disease ( } \% \text { predicted } \\
\text { forced expiratory volume in } 1 \\
\text { second } \geq 50 \% \text { to }<80 \%)\end{array}$ & $6(17 \%)$ \\
\hline $\begin{array}{l}\text { Number of patients using inhaled medicine } \\
\text { for chronic obstructive pulmonary disease }\end{array}$ & $9(26 \%)$ \\
\hline $\begin{array}{l}\text { Number of patients received endobronchial } \\
\text { ultrasonography }\end{array}$ & $24(70 \%)$ \\
\hline Prescribed radiation dose & \\
\hline $60 \mathrm{~Gy} / 30$ fractions/6 weeks once daily & $16(47 \%)$ \\
\hline $\begin{array}{l}64 \mathrm{~Gy} / 40 \text { fractions } / 4 \text { weeks twice daily; } \\
\text { in the morning } 2 \mathrm{~Gy} \text {, with or without } \\
\text { elective nodal irradiation, and in the } \\
\text { evening, } 1.2 \text { Gy with tumor boost }\end{array}$ & $13(38 \%)$ \\
\hline $66 \mathrm{~Gy} / 30$ fractions/6 weeks once daily & $3(8 \%)$ \\
\hline $60 \mathrm{~Gy} / 25$ fractions $/ 5$ weeks once daily & $1(2 \%)$ \\
\hline $\begin{array}{l}40 \mathrm{~Gy} / 20 \text { fractions and } 30 \mathrm{~Gy} / \\
10 \text { fractions once daily }\end{array}$ & $1(2 \%)$ \\
\hline \multicolumn{2}{|l|}{ Prescribed method } \\
\hline Dmean & $32(94 \%)$ \\
\hline D95 & $2(5 \%)$ \\
\hline \multicolumn{2}{|l|}{ Radiotherapy methods } \\
\hline Volumetric modulated arc therapy & $31(91 \%)$ \\
\hline Three-dimensional conformal radiotherapy & $3(8 \%)$ \\
\hline \multicolumn{2}{|l|}{ Elective nodal irradiation } \\
\hline Yes & $12(35 \%)$ \\
\hline No & $22(64 \%)$ \\
\hline \multicolumn{2}{|l|}{ Radiotherapy duration (days) } \\
\hline Median & 41 \\
\hline Range & $15^{\mathrm{a}-61}$ \\
\hline \multicolumn{2}{|l|}{ Planning target volume $(\mathrm{cc})$} \\
\hline Median & 289 \\
\hline Range & 64-1,087 \\
\hline \multicolumn{2}{|l|}{$\begin{array}{l}\text { Lung V5Gy }(\%) \text { (percentage volume of the } \\
\text { lung irradiated more than or equal to } 5 \mathrm{~Gy} \text { ) }\end{array}$} \\
\hline Median & 32 \\
\hline Range & $9-63.5$ \\
\hline \multicolumn{2}{|l|}{$\begin{array}{l}\text { Lung VS5Gy (cc) (absolute volume } \\
\text { of the lung spared from } 5 \text { Gy) }\end{array}$} \\
\hline Median & 2,001 \\
\hline Range & $580-3,998$ \\
\hline \multicolumn{2}{|l|}{ Lung V10Gy (\%) } \\
\hline Median & 20.5 \\
\hline Range & $6.3-36.9$ \\
\hline \multicolumn{2}{|l|}{ Lung V20Gy (\%) } \\
\hline Median & 12.5 \\
\hline Range & 4.4-27 \\
\hline \multicolumn{2}{|l|}{ Lung V30Gy (\%) } \\
\hline Median & 8.4 \\
\hline Range & $1.6-19.9$ \\
\hline \multicolumn{2}{|l|}{ Lung V40Gy (\%) } \\
\hline Median & 4.2 \\
\hline Range & $0.4-14$ \\
\hline
\end{tabular}

Tumor staging was based on TMN classification $7^{\text {th }}$ and $8^{\text {th }}$ edition. The follow-up time was from the day radiotherapy or chemotherapy was started. Any death was counted as an event in the overall survival rate. Any deaths or progression, including distant

\begin{tabular}{|c|c|}
\hline & $\mathrm{N}$ \\
\hline \multicolumn{2}{|l|}{ Lung V50Gy (\%) } \\
\hline Median & 2 \\
\hline Range & $0-8.3$ \\
\hline \multicolumn{2}{|l|}{ Lung V60Gy (\%) } \\
\hline Median & 0 \\
\hline Range & $0-2.4$ \\
\hline \multicolumn{2}{|l|}{ Mean lung dose (Gy) } \\
\hline Median & 7.8 \\
\hline Range & $3.2-14.9$ \\
\hline \multicolumn{2}{|c|}{$\begin{array}{l}\text { Esophagus V10Gy (\%) (percentage volume of the } \\
\text { esophagus irradiated more than or equal to } 10 \mathrm{~Gy} \text { ) }\end{array}$} \\
\hline Median & 45.1 \\
\hline Range & $0-94$ \\
\hline \multicolumn{2}{|l|}{ Esophagus V20Gy (\%) } \\
\hline Median & 35.1 \\
\hline Range & $0-79$ \\
\hline \multicolumn{2}{|l|}{ Esophagus V30Gy (\%) } \\
\hline Median & 29.8 \\
\hline Range & $0-72.8$ \\
\hline \multicolumn{2}{|l|}{ Esophagus V40Gy (\%) } \\
\hline Median & 17.4 \\
\hline Range & $0-66.3$ \\
\hline \multicolumn{2}{|l|}{ Esophagus V50Gy (\%) } \\
\hline Median & 5.1 \\
\hline Range & $0-59.5$ \\
\hline \multicolumn{2}{|l|}{ Esophagus V60Gy (\%) } \\
\hline Median & 0 \\
\hline Range & $0-33$ \\
\hline \multicolumn{2}{|l|}{ Mean esophagus dose (Gy) } \\
\hline Median & 17.5 \\
\hline Range & $1.1-44.6$ \\
\hline \multicolumn{2}{|l|}{ Chemotherapy } \\
\hline \multicolumn{2}{|l|}{$20 \mathrm{mg} / \mathrm{m}^{2}$ days 1,8 triweekly } \\
\hline \multicolumn{2}{|l|}{$40 \mathrm{mg} / \mathrm{m}^{2}$ days $1,8,15,22,29,35$} \\
\hline \multicolumn{2}{|l|}{$100 \mathrm{mg} / \mathrm{m}^{2}$ days $1,8,15$ triweekly } \\
\hline \multicolumn{2}{|l|}{ Chemotherapy cycle } \\
\hline 1 & $2(5 \%)$ \\
\hline 2 & $18(52 \%)$ \\
\hline 3 & $5(14 \%)$ \\
\hline 4 & $6(17 \%)$ \\
\hline 5 & 0 \\
\hline 6 & $3(8 \%)$ \\
\hline \multicolumn{2}{|l|}{ Radiotherapy and chemotherapy timing } \\
\hline Concurrent & $32(94 \%)$ \\
\hline Sequential & $2(5 \%)$ \\
\hline $\begin{array}{l}\text { Number of patients receiving the full } \\
\text { dose of chemotherapy }\end{array}$ & $17(50 \%)$ \\
\hline $\begin{array}{l}\text { Number of patients receiving granulocyte- } \\
\text { colony stimulating factor }\end{array}$ & $7(20 \%)$ \\
\hline
\end{tabular}

AUC: Area under the curve. ${ }^{\text {aThe }}$ patient finished radiotherapy at 24.2 Gy/11 fractions over 15 days because of radiation pneumonitis.

metastases, were counted as an event in the progression-free survival rate. Any progression in the radiotherapy field was counted as an event in the local control rate. Isolated elective nodal failures (i.e., recurrences) were investigated in all thirty-four patients. 
Table II. Univariate and multivariate logistic regression analyses for no use of durvalumab after definitive chemoradiotherapy in non-small cell lung cancer patients.

\begin{tabular}{|c|c|c|c|c|c|c|}
\hline \multirow[t]{2}{*}{ Factors } & \multicolumn{3}{|c|}{ Univariate analyses } & \multicolumn{3}{|c|}{ Multivariate analyses } \\
\hline & $p$-Value & $\begin{array}{l}\text { Odds } \\
\text { ratio }\end{array}$ & $\begin{array}{l}95 \% \text { confidence } \\
\text { interval }\end{array}$ & $p$-Value & $\begin{array}{l}\text { Odds } \\
\text { ratio }\end{array}$ & $\begin{array}{l}95 \% \text { confidence } \\
\text { interval }\end{array}$ \\
\hline $\begin{array}{l}\text { Chronic obstructive pulmonary disease } \\
\text { (vs. no chronic obstructive pulmonary disease) }\end{array}$ & 0.003 & 18.37 & $2.74-122.94$ & 0.022 & 17.25 & $1.51-196.64$ \\
\hline Elective nodal irradiation ( $v s$. no elective nodal irradiation) & 0.005 & 14 & $2.19-89.21$ & 0.016 & 19.81 & $1.98-252.82$ \\
\hline Forced expiratory volume in 1 second (1) & 0.031 & 0.14 & $0.024-0.83$ & Not included & & \\
\hline Forced expiratory volume in 1 second $\%$ & 0.007 & 0.89 & $0.82-0.96$ & Not included & & \\
\hline$\%$ predicted forced expiratory volume in $1 \mathrm{sec}$ & 0.019 & 0.93 & $0.88-0.98$ & Not included & & \\
\hline $\begin{array}{l}\text { Use of inhaled medicine for chronic obstructive pulmonary } \\
\text { disease ( } v s . \text { no use of inhaled medicine) }\end{array}$ & 0.03 & 6.56 & $1.2-35.73$ & Not included & & \\
\hline $\begin{array}{l}\text { Radiation pneumonitis } \geq \text { Grade } 2 \text { ( } v s \text {. Grade } 1 \\
\text { or no radiation pneumonitis) }\end{array}$ & 0.016 & 8 & $1.46-43.67$ & Not included & & \\
\hline $\begin{array}{l}\text { Radiation esophagitis } \geq \text { Grade } 2 \text { (vs. Grade } 1 \\
\text { or no radiation esophagitis) }\end{array}$ & 0.045 & 12 & $1.05-136.79$ & Not included & & \\
\hline Age & 0.094 & & & 0.404 & & \\
\hline Male ( $v s$. female) & 0.24 & & & Not included & & \\
\hline Performance status $1(v s .0)$ & 0.28 & & & Not included & & \\
\hline $\begin{array}{l}\text { Location of primary site in upper or } \\
\text { middle lobe ( } v s . \text { in lower lobe) }\end{array}$ & 0.94 & & & Not included & & \\
\hline $\mathrm{T} 3$ or $\mathrm{T} 4(v s . \mathrm{T} 0$ or $\mathrm{T} 1$ or $\mathrm{T} 2)$ & 0.65 & & & Not included & & \\
\hline $\mathrm{N} 2$ or $\mathrm{N} 3(v s . \mathrm{N} 0$ or $\mathrm{N} 1)$ & 0.65 & & & Not included & & \\
\hline Stage III ( $v s$. Stage I or II) & 0.67 & & & Not included & & \\
\hline $\begin{array}{l}\text { Adenocarcinoma ( } v s . \text { squamous cell carcinoma or } \\
\text { pleomorphic carcinoma) }\end{array}$ & 0.17 & & & Not included & & \\
\hline $\begin{array}{l}\text { Epidermal growth factor receptor mutation } \\
\text { (vs. no epidermal growth factor receptor mutation) }\end{array}$ & 0.44 & & & Not included & & \\
\hline $\begin{array}{l}\text { Anaplastic lymphoma kinase fusion gene } \\
\text { (vs. no anaplastic lymphoma kinase fusion gene) }\end{array}$ & 0.37 & & & Not included & & \\
\hline $\begin{array}{l}\text { C-ros oncogene } 1 \text { fusion gene } \\
\text { (vs. no c-ros oncogene } 1 \text { fusion gene) }\end{array}$ & 0.86 & & & Not included & & \\
\hline $\begin{array}{l}\text { Programmed cell death } 1 \text { ligand } 1 \\
\text { expression high }(\geq 50 \%)(v s . \text { low }<50 \%)\end{array}$ & 0.16 & & & Not included & & \\
\hline $\begin{array}{l}\text { Positron emission tomography standardized } \\
\text { maximum uptake value }\end{array}$ & 0.29 & & & Not included & & \\
\hline Brinkman index & 0.49 & & & Not included & & \\
\hline Alcohol history ( $v s$. no alcohol history) & 0.94 & & & Not included & & \\
\hline Body mass index & 0.086 & & & Not included & & \\
\hline Pulmonary emphysema ( $v s$. no pulmonary emphysema) & 0.34 & & & Not included & & \\
\hline Interstitial pneumonia ( $v s$. no interstitial pneumonia) & 0.99 & & & Not included & & \\
\hline Asthma ( $v s$. no asthma) & 0.45 & & & Not included & & \\
\hline Vital capacity (1) & 0.23 & & & Not included & & \\
\hline$\%$ vital capacity & 0.24 & & & Not included & & \\
\hline $\begin{array}{l}\text { Intermediate chronic obstructive pulmonary disease } \\
\text { ( } v s . \text { no chronic obstructive pulmonary disease or } \\
\text { mild chronic obstructive pulmonary disease) }\end{array}$ & 0.16 & & & Not included & & \\
\hline Diabetes mellitus ( $v s$. no diabetes mellitus) & 0.67 & & & Not included & & \\
\hline White blood cell count $/ \mathrm{mm}^{3}$ & 0.61 & & & Not included & & \\
\hline Neutrophil cell count $/ \mathrm{mm}^{3}$ & 0.45 & & & Not included & & \\
\hline Hemoglobin (g/dl) & 0.13 & & & Not included & & \\
\hline Platelet cell count $/ \mathrm{mm}^{3}$ & 0.4 & & & Not included & & \\
\hline C-reactive protein $(\mathrm{mg} / \mathrm{dl})$ & 0.37 & & & Not included & & \\
\hline Creatinine clearance $(\mathrm{ml} / \mathrm{min})$ & 0.077 & & & Not included & & \\
\hline Carcinoembryonic antigen $(\mathrm{ng} / \mathrm{ml})$ & 0.24 & & & Not included & & \\
\hline Cytokeratin 19 fragment (ng/ml) & 0.3 & & & Not included & & \\
\hline Sialylated carbohydrate antigen KL-6 $(\mathrm{U} / \mathrm{ml})$ & 0.45 & & & Not included & & \\
\hline Post-operative recurrences (vs. new cases) & 0.42 & & & Not included & & \\
\hline
\end{tabular}


Table II. Continued

\begin{tabular}{|c|c|c|c|c|c|c|}
\hline \multirow[t]{2}{*}{ Factors } & \multicolumn{3}{|c|}{ Univariate analyses } & \multicolumn{3}{|c|}{ Multivariate analyses } \\
\hline & $p$-Value & $\begin{array}{l}\text { Odds } \\
\text { ratio }\end{array}$ & $\begin{array}{l}\text { 95\% confidence } \\
\text { interval }\end{array}$ & $p$-Value & $\begin{array}{l}\text { Odds } \\
\text { ratio }\end{array}$ & $\begin{array}{l}95 \% \text { confidence } \\
\text { interval }\end{array}$ \\
\hline Use of carboplatin ( $v s$. use of cisplatin) & 0.057 & & & Not included & & \\
\hline $\begin{array}{l}\text { No use of full dose of chemotherapy } \\
\text { ( } v s . \text { use of full dose of chemotherapy) }\end{array}$ & 0.99 & & & Not included & & \\
\hline Number of chemotherapy cycles & 0.52 & & & Not included & & \\
\hline $\begin{array}{l}\text { Use of granulocyte-colony stimulating factor } \\
\text { ( } v s . \text { no use of granulocyte-colony stimulating factor) }\end{array}$ & 0.42 & & & Not included & & \\
\hline $\begin{array}{l}\text { Axillary temperature of } \geq 37.5^{\circ} \mathrm{C} \text { during } \\
\text { chemoradiotherapy }\left(v s .<37.5^{\circ} \mathrm{C}\right)\end{array}$ & 0.76 & & & Not included & & \\
\hline Febrile neutropenia ( $v s$. no febrile neutropenia) & 0.99 & & & Not included & & \\
\hline $\begin{array}{l}\text { White blood cells decreased } \geq \text { Grade } 3 \text { (vs. Grade } 1 \\
\text { or } 2 \text { or no white blood cells decreased) }\end{array}$ & 0.34 & & & Not included & & \\
\hline Neutropenia $\geq$ Grade 3 (vs. Grade 1 or 2 or no neutropenia) & 0.34 & & & Not included & & \\
\hline Anemia $\geq$ Grade 2 (vs. Grade 1 or no anemia) & 0.91 & & & Not included & & \\
\hline Thrombocytopenia $\geq$ Grade 1 ( $v s$. no thrombocytopenia) & 0.81 & & & Not included & & \\
\hline $\begin{array}{l}\text { Twice-daily thoracic radiotherapy } \\
\text { (vs. once-daily thoracic radiotherapy) }\end{array}$ & 0.26 & & & Not included & & \\
\hline $\begin{array}{l}\text { Volumetric modulated arc therapy } \\
\text { (vs. three-dimensional conformal radiotherapy) }\end{array}$ & 0.77 & & & Not included & & \\
\hline Radiotherapy duration (days) & 0.41 & & & Not included & & \\
\hline Planning target volume (cc) & 0.45 & & & Not included & & \\
\hline $\begin{array}{l}\text { Lung V5Gy (\%) (percentage volume of the } \\
\text { lung irradiated more than or equal to } 5 \mathrm{~Gy} \text { ) }\end{array}$ & 0.58 & & & Not included & & \\
\hline $\begin{array}{l}\text { Lung VS5Gy (cc) (absolute volume } \\
\text { of the lung spared from } 5 \mathrm{~Gy} \text { ) }\end{array}$ & 0.068 & & & Not included & & \\
\hline Lung V10Gy (\%) & 0.59 & & & Not included & & \\
\hline Lung V20Gy (\%) & 0.74 & & & Not included & & \\
\hline Lung V30Gy (\%) & 0.93 & & & Not included & & \\
\hline Lung V40Gy (\%) & 0.95 & & & Not included & & \\
\hline Lung V50Gy (\%) & 0.98 & & & Not included & & \\
\hline Lung V60Gy (\%) & 0.081 & & & Not included & & \\
\hline Mean lung dose (Gy) & 0.58 & & & Not included & & \\
\hline $\begin{array}{l}\text { Esophagus V10Gy (\%) (percentage volume of the } \\
\text { esophagus irradiated more than or equal to } 10 \mathrm{~Gy} \text { ) }\end{array}$ & 0.76 & & & Not included & & \\
\hline Esophagus V20Gy (\%) & 0.58 & & & Not included & & \\
\hline Esophagus V30Gy (\%) & 0.46 & & & Not included & & \\
\hline Esophagus V40Gy (\%) & 0.28 & & & Not included & & \\
\hline Esophagus V50Gy (\%) & 0.71 & & & Not included & & \\
\hline Esophagus V60Gy (\%) & 0.67 & & & Not included & & \\
\hline Mean esophagus dose (Gy) & 0.55 & & & Not included & & \\
\hline
\end{tabular}

Values in italics are statistically significant $(p<0.05)$. Multivariate analyses were done as reference only because the study size included only 34 cases, and the result might be unstable.

Radiotherapy. Volumetric modulated arc therapy (VMAT) with mainly Dmean prescription was generally performed. All patients received four-dimensional or three-dimensional CT simulation. Clinical target volume (CTV) 1 was typically a $0.5-\mathrm{cm}$ expansion of the gross tumor volume including the primary tumor and metastatic lymph nodes; planning target volume (PTV) 1 was then a $0.5-\mathrm{cm}$ expansion of CTV1. Then, the radiation oncologist determined whether CTV2 should include elective hilar, mediastinal, or supraclavicular lymph nodes plus CTV1 based on the information of the patient, tumor, and treatment. This protocol is called elective nodal irradiation (ENI) and is intended to treat potential metastatic lymph nodes. The target of the elective nodal area was delineated based on the Japan Lung Cancer Society-Japanese Society for Radiation Oncology consensus-based computed tomographic atlas for defining regional lymph node stations in radiotherapy for lung cancer $(3,4)$. The total radiation dose of 60 $\mathrm{Gy} / 30$ fractions/6 weeks once daily was mainly administered to the patients. Alternatively, a more aggressive dose of $64 \mathrm{~Gy} / 40$ fractions/4 weeks twice daily (accelerated hyperfractionated thoracic radiotherapy) was given (5-7); in the morning, 2 Gy with or without ENI, and in the evening, 1.2 Gy with tumor boost radiotherapy were 
Table III. Frequency and reasons for not receiving durvalumab within 45 days after chemoradiotherapy.

Number of patients who did not

Nine of 34 patients $(26 \%)$ receive durvalumab within

45 days after radiotherapy

The reasons for not receiving durvalumab

Radiation pneumonitis ${ }^{\mathrm{a}}$

Radiation esophagitis ${ }^{b}$

Progression of disease

Death from heart attack

Leg bone fracture

Not coming to the hospital

Five of 10 reasons $(50 \%)$

One of 10 reasons $(10 \%)$

One of 10 reasons $(10 \%)$

One of 10 reasons $(10 \%)$

One of 10 reasons $(10 \%)$

One of 10 reasons $(10 \%)$

a,b One patient had two reasons.

administered to other patients. ENI was performed for a median of 40 Gy/20 fractions/4 weeks. ENI was preferably adopted in good performance patients or patients in whom the tumor was located in the upper lobe, which requires a smaller lung dose compared to those with a primary location in the lower lobe.

Chemotherapy and immunotherapy. The chemotherapy regimen mainly consisted of cisplatin and vinorelbine or carboplatin and paclitaxel. Cisplatin $\left(80 \mathrm{mg} / \mathrm{m}^{2}\right)$ on day 1 combined with vinorelbine $\left(20 \mathrm{mg} / \mathrm{m}^{2}\right)$ on days 1 and 8 in 3- to 4 -week intervals were delivered concurrently with radiotherapy. In patients aged $>75$ years or those with a low performance status, low renal function (creatinine clearance $<60 \mathrm{ml} / \mathrm{min}$ ), or other severe complications, the second choice for concurrent chemotherapy consisted of weekly carboplatin (area under the curve $=2)$ plus paclitaxel $\left(40 \mathrm{mg} / \mathrm{m}^{2}\right)$, which were administered concurrently with radiotherapy. Another regimen, carboplatin (area under the curve $=6$ ) on day 1 combined with nab-paclitaxel $\left(100 \mathrm{mg} / \mathrm{m}^{2}\right)$ on days 1,8 , and 15 delivered concurrently with radiotherapy or pre-radiotherapy, was used. After chemoradiotherapy, durvalumab $(10 \mathrm{mg} / \mathrm{kg})$ every 2 weeks for up to 12 months was given if possible.

Statistical analysis. The endpoint of this study was that durvalumab was given to patients within 45 days after chemoradiotherapy. Although 42 days ( 6 weeks) was the longest interval between the end of chemoradiotherapy and start of durvalumab in a previous phase III study $(1,2)$, the one and a half-month (45 days) interval was adopted in this daily clinical practice study. Univariate logistic regression analyses were performed for 71 clinical and therapeutic factors. Then, selected factors were entered into the multivariate logistic regression analyses. However, the results of multivariate analyses are shown in Table II for reference only, because the sample size was 34 cases, and thus, results might be unstable. Statistical analyses were performed using SPSS, version 24.0 (IBM, Armonk, NY, USA). The 1-year overall survival, 1-year local control, and 1-year progression-free survival rates were estimated, and percentages were calculated with the Kaplan-Meier method.

\section{Results}

The median follow-up time was 13 months (range=0-17 months). The 1-year overall survival rate was $89 \%$
Table IV. Adverse events due to chemoradiotherapy.

\begin{tabular}{|c|c|}
\hline Adverse events & $\mathrm{N}$ \\
\hline \multicolumn{2}{|l|}{ Pneumonitis } \\
\hline Grade 1 & $14(41 \%)$ \\
\hline Grade 2 & $10(29 \%)$ \\
\hline Grade 3 & 0 \\
\hline Grade 4 & $1(2 \%)$ \\
\hline Grade 5 & 0 \\
\hline \multicolumn{2}{|l|}{ Esophagitis } \\
\hline Grade 1 & $14(41 \%)$ \\
\hline Grade 2 & $1(2 \%)$ \\
\hline Grade 3 & $3(8 \%)$ \\
\hline Grade 4 & 0 \\
\hline Grade 5 & 0 \\
\hline \multicolumn{2}{|l|}{ Febrile neutropenia } \\
\hline Grade 3 & $2(5 \%)$ \\
\hline Grade 4 & 0 \\
\hline Grade 5 & 0 \\
\hline \multicolumn{2}{|l|}{ White blood cells decreased } \\
\hline Grade 1 & $2(5 \%)$ \\
\hline Grade 2 & $10(29 \%)$ \\
\hline Grade 3 & $16(47 \%)$ \\
\hline Grade 4 & $2(5 \%)$ \\
\hline Grade 5 & 0 \\
\hline \multicolumn{2}{|l|}{ Neutropenia } \\
\hline Grade 1 & $2(5 \%)$ \\
\hline Grade 2 & $13(38 \%)$ \\
\hline Grade 3 & $9(26 \%)$ \\
\hline Grade 4 & $3(8 \%)$ \\
\hline Grade 5 & 0 \\
\hline \multicolumn{2}{|l|}{ Anemia } \\
\hline Grade 1 & $24(70 \%)$ \\
\hline Grade 2 & $8(23 \%)$ \\
\hline Grade 3 & 0 \\
\hline Grade 4 & 0 \\
\hline Grade 5 & 0 \\
\hline \multicolumn{2}{|l|}{ Thrombocytopenia } \\
\hline Grade 1 & $19(55 \%)$ \\
\hline Grade 2 & $1(2 \%)$ \\
\hline Grade 3 & 0 \\
\hline Grade 4 & 0 \\
\hline Grade 5 & 0 \\
\hline $\begin{array}{l}\text { Number of patients with an axillary temperature } \\
\text { of } \geq 37.5^{\circ} \mathrm{C} \text { during chemoradiotherapy }\end{array}$ & $10(29 \%)$ \\
\hline
\end{tabular}

(range $=79-100 \%)$. The 1-year progression-free survival rate was $72 \%($ range $=56-87 \%)$. The 1 -year local control rate was $76 \%$ (range $=60-92 \%$ ). No isolated elective nodal failures were found in all thirty-four patients.

Twenty-five of 34 (73\%) patients received immunotherapy after a median of 8 days (range $=1-45$ days) from the end of chemoradiotherapy. The reasons for not receiving immunotherapy are shown in Table III. The most frequent reason for not receiving immunotherapy was radiation pneumonitis, which accounted for 50\% (5/10 reasons). Adverse events including hematologic toxicities due to 
Table V. Univariate logistic regression analyses for not receiving durvalumab after definitive chemoradiotherapy in thirty-four non-small cell lung cancer patients.

\begin{tabular}{|c|c|c|c|}
\hline \multirow[t]{2}{*}{ Factors } & \multicolumn{3}{|c|}{ Univariate analyses } \\
\hline & $p$-Value & $\begin{array}{l}\text { Odds } \\
\text { ratio }\end{array}$ & $\begin{array}{l}95 \% \text { confidence } \\
\text { interval }\end{array}$ \\
\hline Chronic obstructive pulmonary disease ( $v s$. no chronic obstructive pulmonary disease) & 0.003 & 18.37 & 2.74-122.94 \\
\hline Elective nodal irradiation ( $v s$. no elective nodal irradiation) & 0.005 & 14 & $2.19-89.21$ \\
\hline Forced expiratory volume in 1 second (1) & 0.031 & 0.14 & $0.024-0.83$ \\
\hline Forced expiratory volume in 1 second $\%$ & 0.007 & 0.89 & $0.82-0.96$ \\
\hline$\%$ predicted forced expiratory volume in 1 second & 0.019 & 0.93 & $0.88-0.98$ \\
\hline $\begin{array}{l}\text { Use of inhaled medicine for chronic obstructive pulmonary } \\
\text { disease ( } v s . \text { no use of inhaled medicine) }\end{array}$ & 0.03 & 6.56 & $1.2-35.73$ \\
\hline Radiation pneumonitis $\geq$ Grade 2 ( $v s$. Grade 1 or no radiation pneumonitis) & 0.016 & 8 & $1.46-43.67$ \\
\hline Radiation esophagitis $\geq$ Grade 2 ( $v s$. Grade 1 or no radiation esophagitis) & 0.045 & 12 & $1.05-136.79$ \\
\hline Age & 0.094 & & \\
\hline Male (vs. female) & 0.24 & & \\
\hline Performance status 1 (vs. 0) & 0.28 & & \\
\hline Location of primary site in upper or middle lobe ( $v s$. in lower lobe) & 0.94 & & \\
\hline $\mathrm{T} 3$ or $\mathrm{T} 4$ (vs. $\mathrm{T} 0$ or $\mathrm{T} 1$ or $\mathrm{T} 2)$ & 0.65 & & \\
\hline $\mathrm{N} 2$ or $\mathrm{N} 3(v s . \mathrm{N} 0$ or $\mathrm{N} 1)$ & 0.65 & & \\
\hline Stage III ( $v s$. Stage I or II) & 0.67 & & \\
\hline Adenocarcinoma ( $v s$. squamous cell carcinoma or pleomorphic carcinoma) & 0.17 & & \\
\hline Epidermal growth factor receptor mutation ( $v s$. no epidermal growth factor receptor mutation) & 0.44 & & \\
\hline Anaplastic lymphoma kinase fusion gene ( $v s$. no anaplastic lymphoma kinase fusion gene) & 0.37 & & \\
\hline C-ros oncogene 1 fusion gene ( $v s$. no c-ros oncogene 1 fusion gene) & 0.86 & & \\
\hline Programmed cell death 1 ligand 1 expression high $(\geq 50 \%)(v s$. low $<50 \%)$ & 0.16 & & \\
\hline Positron emission tomography standardized maximum uptake value & 0.29 & & \\
\hline Brinkman index & 0.49 & & \\
\hline Alcohol history (vs. no alcohol history) & 0.94 & & \\
\hline Body mass index & 0.086 & & \\
\hline Pulmonary emphysema ( $v s$. no pulmonary emphysema) & 0.34 & & \\
\hline Interstitial pneumonia (vs. no interstitial pneumonia) & 0.99 & & \\
\hline Asthma (vs. no asthma) & 0.45 & & \\
\hline Vital capacity (1) & 0.23 & & \\
\hline$\%$ vital capacity & 0.24 & & \\
\hline $\begin{array}{l}\text { Intermediate chronic obstructive pulmonary disease ( } v s . \text { no chronic obstructive } \\
\text { pulmonary disease or mild chronic obstructive pulmonary disease) }\end{array}$ & 0.16 & & \\
\hline Diabetes mellitus ( $v s$. no diabetes mellitus) & 0.67 & & \\
\hline White blood cell count $/ \mathrm{mm}^{3}$ & 0.61 & & \\
\hline Neutrophil cell count $/ \mathrm{mm}^{3}$ & 0.45 & & \\
\hline Hemoglobin $(\mathrm{g} / \mathrm{dl})$ & 0.13 & & \\
\hline Platelet cell count $/ \mathrm{mm}^{3}$ & 0.4 & & \\
\hline C-reactive protein $(\mathrm{mg} / \mathrm{dl})$ & 0.37 & & \\
\hline Creatinine clearance $(\mathrm{ml} / \mathrm{min})$ & 0.077 & & \\
\hline Carcinoembryonic antigen $(\mathrm{ng} / \mathrm{ml})$ & 0.24 & & \\
\hline Cytokeratin 19 fragment $(\mathrm{ng} / \mathrm{ml})$ & 0.3 & & \\
\hline Sialylated carbohydrate antigen KL-6 (U/ml) & 0.45 & & \\
\hline Post-operative recurrences ( $v s$. new cases) & 0.42 & & \\
\hline Use of Carboplatin (vs. use of cisplatin) & 0.057 & & \\
\hline No use of full dose of chemotherapy (vs. use of full dose of chemotherapy) & 0.99 & & \\
\hline Number of chemotherapy cycles & 0.52 & & \\
\hline Use of granulocyte-colony stimulating factor ( $v s$. no use of granulocyte-colony stimulating factor) & 0.42 & & \\
\hline Axillary temperature of $\geq 37.5^{\circ} \mathrm{C}$ during chemoradiotherapy $\left(v s .<37.5^{\circ} \mathrm{C}\right)$ & 0.76 & & \\
\hline Febrile neutropenia ( $v s$. no febrile neutropenia) & 0.99 & & \\
\hline White blood cells decreased $\geq$ Grade 3 ( $v s$. Grade 1 or 2 or no white blood cells decreased) & 0.34 & & \\
\hline Neutropenia $\geq$ Grade 3 ( $v s$. Grade 1 or 2 or no neutropenia) & 0.34 & & \\
\hline Anemia $\geq$ Grade 2 ( $v s$. Grade 1 or no anemia) & 0.91 & & \\
\hline Thrombocytopenia $\geq$ Grade 1 (vs. no thrombocytopenia) & 0.81 & & \\
\hline Twice-daily thoracic radiotherapy (vs. once-daily thoracic radiotherapy) & 0.26 & & \\
\hline
\end{tabular}


Table V. Continued

\begin{tabular}{ll}
\hline Factors & \multicolumn{1}{c}{ Univariate analyses } \\
\cline { 2 - 2 } & $\begin{array}{c}\text { Odds } \\
\text { ratio }\end{array}$ \\
\hline Volumetric modulated arc therapy ( $v$ s. three-dimensional conformal radiotherapy) & 0.77 \\
Radiotherapy duration (days) & 0.41 \\
Planning target volume (cc) & 0.45 \\
Lung V5Gy (\%) (percentage volume of the lung irradiated more than or equal to 5 Gy) & 0.58 \\
Lung VS5Gy (cc) (absolute volume of the lung spared from 5 Gy) & 0.068 \\
Lung V10Gy (\%) & 0.59 \\
Lung V20Gy (\%) & 0.74 \\
Lung V30Gy (\%) & 0.93 \\
Lung V40Gy (\%) & 0.95 \\
Lung V50Gy (\%) & 0.98 \\
Lung V60Gy (\%) & 0.081 \\
Mean lung dose (Gy) & 0.58 \\
Esophagus V10Gy (\%) (percentage volume of the esophagus irradiated more than or equal to 10 Gy) & 0.76 \\
Esophagus V20Gy $(\%)$ & 0.58 \\
Esophagus V30Gy (\%) & 0.46 \\
Esophagus V40Gy (\%) & 0.28 \\
Esophagus V50Gy (\%) & 0.71 \\
Esophagus V60Gy (\%) & 0.67 \\
Mean esophagus dose (Gy) & 0.55
\end{tabular}

Values in italics are statistically significant $(p<0.05)$.

chemoradiotherapy are shown in Table IV. Radiation pneumonitis $\geq$ Grade 2 and radiation esophagitis $\geq$ Grade 2 were found in $32 \%$ ( $11 / 34$ cases) and $11 \%$ (4/34 cases), respectively. The eight significant factors in univariate analysis that affected administration of immunotherapy are listed in Table $\mathrm{V}$ and are as follows: chronic obstructive pulmonary disease (COPD)+, ENI+, using inhaled medicine for COPD, forced expiratory volume in 1 second (FEV1), FEV1\%, \%FEV1, radiation pneumonitis $\geq$ Grade 2 , and radiation esophagitis $\geq$ Grade 2 . COPD was defined as FEV $1 \%<70 \%$ in this study. Lung dose parameters including lung VS5Gy (cc) (absolute volume of the lung spared from 5 Gy) (8) were not significant factors. To avoid multicollinearity, COPD+, ENI+, and age, were included in the multivariate logistic regression analyses. Although age was not a significant factor in univariate analysis, age was important in clinical practice. After multivariate logistic regression analysis, two significant factors affecting immunotherapy were identified as COPD+ and ENI+ (for reference only; Table II). The actual rates of immunotherapy based on COPD (+ or -) or ENI (+ or - ) are shown in Table VI. The rate of immunotherapy was $100 \%$ (17/17 cases) in the COPD- and ENI- group, and $16 \%$ (1/6 cases) in the COPD+ and ENI+ group. The choice of ENI area and the background of the patients are shown in Table VII. The comparison of lung dose parameters between the group that
Table VI. The rate of use of durvalumab with or without chronic obstructive pulmonary diseases (COPD), and with or without elective nodal irradiation (ENI) after definitive chemoradiotherapy in thirty-four non-small cell lung cancer patients.

\begin{tabular}{lccc}
\hline & ENI $(+)$ & ENI $(-)$ & Total \\
\hline COPD $(+)$ & $1 / 6(16 \%)$ & $3 / 5(60 \%)$ & $4 / 11(36 \%)$ \\
COPD $(-)$ & $4 / 6(66 \%)$ & $17 / 17(100 \%)$ & $21 / 23(91 \%)$ \\
Total & $5 / 12(41 \%)$ & $20 / 22(90 \%)$ & $25 / 34(73 \%)$ \\
\hline
\end{tabular}

received durvalumab and the group that did not is shown in Table VIII. No significant differences were found in any of the average lung doses between the two groups.

Five patients could not receive immunotherapy because of radiation pneumonitis. Four of these five patients with radiation pneumonitis received ENI. Therefore, for these four patients, the virtual radiation therapy planning was done without ENI (i.e., without PTV2) in order to prove the reduction of the lung dose parameters using their past radiation therapy planning CT with fusion of diagnostic CT images of emergence of their radiation pneumonitis. The radiation pneumonitis area on diagnostic CT with fusion on the radiation therapy planning CT was delineated, and a virtual radiation therapy plan without ENI was made. For all four patients, reduction of the dose in 


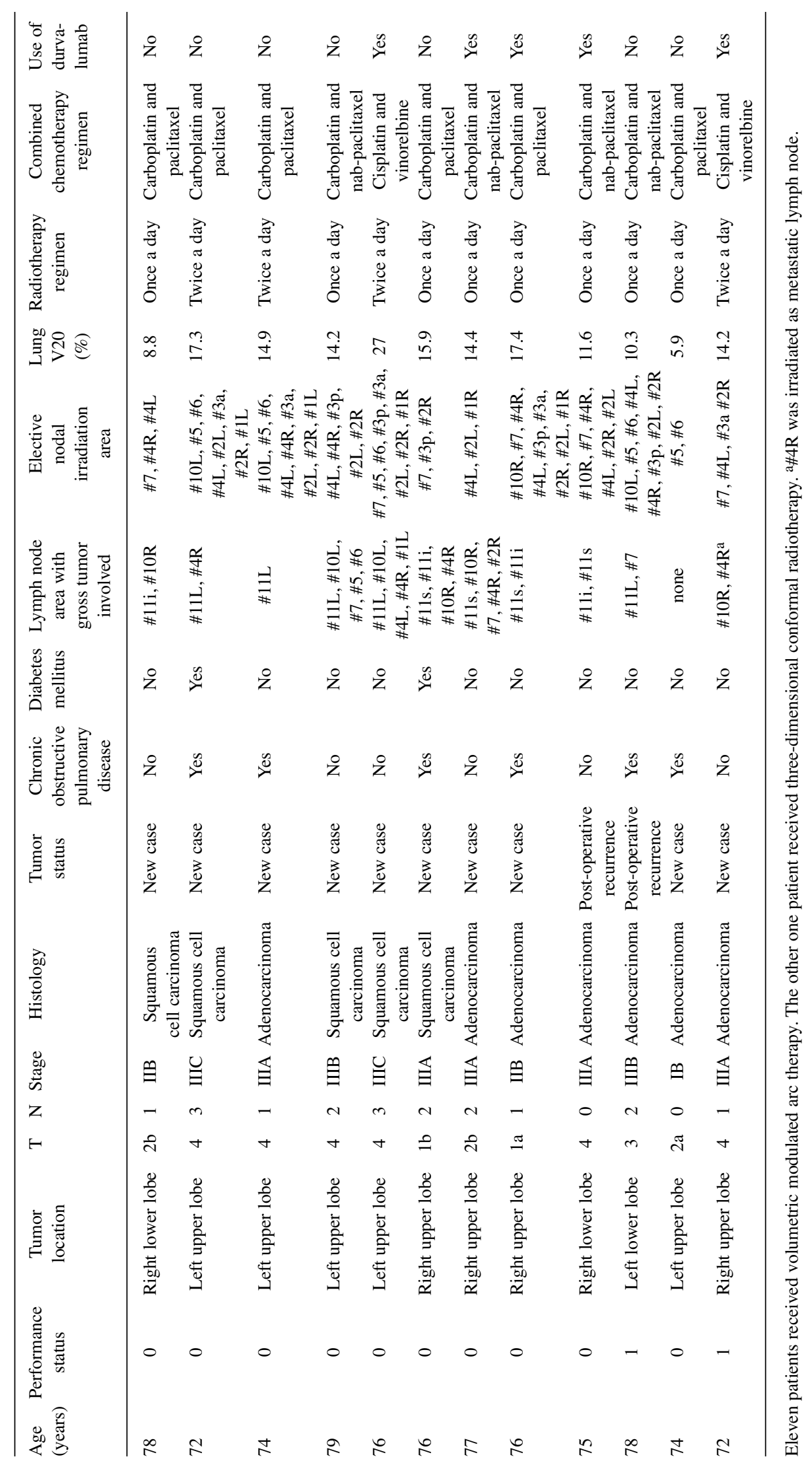


Table VIII. Comparison of dosimetric parameters of lung dose between group that used durvalumab and group that did not.

\begin{tabular}{|c|c|c|c|}
\hline Lung dose & $\begin{array}{l}\text { Use of durvalumab } \\
\qquad(\mathrm{N}=25)\end{array}$ & $\begin{array}{l}\text { No use of durvalumab } \\
\qquad(\mathrm{N}=9)\end{array}$ & $p$-Value \\
\hline Average V5Gy (\%) (percentage volume of the lung irradiated more than or equal to $5 \mathrm{~Gy}$ ) & 34 & 36.8 & 0.523 \\
\hline Average VS5Gy (cc) (absolute volume of the lung spared from $5 \mathrm{~Gy}$ ) & 1847 & 2383 & 0.093 \\
\hline Average V10Gy (\%) & 21.1 & 22.7 & 0.536 \\
\hline Average V20Gy (\%) & 12.4 & 13.1 & 0.742 \\
\hline Average V30Gy (\%) & 8.4 & 8.6 & 0.941 \\
\hline Average V40Gy (\%) & 5.3 & 5.2 & 0.964 \\
\hline Average V50Gy (\%) & 2.6 & 2.6 & 0.984 \\
\hline Average V60Gy (\%) & 0.2 & 0.6 & 0.191 \\
\hline Average mean lung dose (Gy) & 7.9 & 8.5 & 0.555 \\
\hline
\end{tabular}

the radiation pneumonitis area, lung dose parameters, and esophagus dose parameters could be achieved by maintaining the effective dose to PTV1 (i.e., the primary tumor and metastatic lymph nodes) (Table IX).

\section{Discussion}

Although our study size was small, this study calculated the actual rate of immunotherapy, analyzed clinical and dosimetric factors, and demonstrated that ENI and COPD affected whether NSCLC patients did or did not receive immunotherapy after definitive chemoradiotherapy. Radiation pneumonitis was the most common reason for not receiving immunotherapy. Virtual radiation therapy plan without ENI in patients treated with ENI who could not receive immunotherapy because of their radiation pneumonitis, achieved a reduction in the dose parameters of the lung. The results showed the possibility of palliation for their radiation pneumonitis without ENI. Although the follow-up time was short, no isolated elective nodal failures were found in all patients in the current study.

ENI has not been recommended for NSCLC in the guidelines of the European Organization for Research and Treatment of Cancer (9). In the International Atomic Energy Agency report (10), the clinical value of ENI was uncertain. The clinical trial Radiation Therapy Oncology Group 0617 did not permit ENI (11). On the other hand, ENI was regarded as an alternative standard therapy for locally advanced lung cancer in the Japanese radiation therapy planning guideline in 2016 (12) when immunotherapy was not available after definitive chemoradiotherapy. Although ENI has been used traditionally (13), its effectiveness has not been established. Yuan et al. reported that high-dose involved field irradiation (IFI) achieves a better overall response rate (90\% vs. 79\%, $p=0.032)$, better 5-year local control rate $(51 \%$ vs. $36 \%, p=0.032$ ), and better 2 -year overall survival rate (39.4\% vs. 25.6\%, $p=0.048)$ than low-dose ENI in a randomized trial of 200 patients (14). The radiation pneumonitis rate in the IFI group was also lower than that in the ENI group (17\% vs. 29\%, p=0.044). Topkan et al. reported that isolated elective nodal failures were present in $2.5 \%$ of the ENI group (21/844 cases) vs. $2.1 \%$ (3/143 cases) of the IFI group (15). They also reported that the overall survival rate (22.3 vs. 23.7 months, $p=0.47)$, locoregional progression-free survival rate (12.6 vs. 13.2 months, $p=0.58)$, and progression-free survival rate (10.7 vs. 10.4 months, $p=0.82$ ) were not significantly different between the two groups. In a meta-analysis, Li et al. reported no significant difference in the incidence of elective nodal failure between the IFI and ENI groups in three randomized controlled trials and three cohort studies (16). Schild et al. reported that ENI is associated with worse survival than IFI (median survival 16 vs. 24 months, $p=0.002$ ) in a pooled analysis of 16 cooperative group trials involving 3,600 patients (17).

Staging accuracy was achieved with positron emission tomography/CT and endobronchial ultrasonography. The progressed supportive care recommendation for NSCLC patients receiving chemoradiotherapy has been described (18). Radiotherapy technology was developed by four-dimensional CT, intensity modulated radiation therapy (IMRT), VMAT, and daily cone-beam CT. Although ENI compensates for the developing staging accuracy or radiation technology, omission of ENI for decreasing radiation-induced adverse events including pneumonitis is a reasonable idea for introducing immunotherapy after chemoradiotherapy in this era. Although the radiation oncologist should decrease the normal tissue dose including the dose to the lung and concentrate the effective dose on the gross tumor, they should remember which lymph nodes lung cancers tend to spread. This way of thinking may encourage another future study such as a study that includes post-operative radiotherapy.

Around 39-62\% of lung cancer patients also have COPD $(19,20)$. In the current study, one-third of NSCLC patients had COPD, and most of them received an acting antimuscarinic antagonist, acting beta agonist, or inhaled corticosteroid. COPD and radiation pneumonitis are 
Table IX. Comparison of dosimetric factors between virtual radiation therapy plans without elective nodal irradiation (ENI) and actual radiation therapy plans with ENI in the four radiation pneumonitis patients who could not receive durvalumab $(N=4)$.

\begin{tabular}{|c|c|c|c|}
\hline & Dosimetric factors & $\begin{array}{l}\text { Actual radiation } \\
\text { therapy plan } \\
\text { with ENI }\end{array}$ & $\begin{array}{l}\text { Virtual radiation } \\
\text { therapy plan } \\
\text { without ENI }\end{array}$ \\
\hline \multirow[t]{18}{*}{ Patient 1} & Planning target volume (cc) & 501 & 262 \\
\hline & Lung V5Gy (\%) (percentage volume of the lung irradiated more than or equal to $5 \mathrm{~Gy}$ ) & 42.4 & 32.1 \\
\hline & Lung VS5Gy (cc) (absolute volume of the lung spared from $5 \mathrm{~Gy}$ ) & 2299 & 2708 \\
\hline & Lung V10Gy (\%) & 27.6 & 20.2 \\
\hline & Lung V20Gy (\%) & 14.2 & 12.3 \\
\hline & Lung V30Gy (\%) & 9.3 & 8.1 \\
\hline & Lung V40Gy (\%) & 5.7 & 5.4 \\
\hline & Lung V50Gy (\%) & 1.6 & 1.3 \\
\hline & Lung V60Gy (\%) & 0 & 0 \\
\hline & Mean lung dose (Gy) & 10.3 & 7.7 \\
\hline & Mean dose of radiation pneumonitis area (Gy) & 19.6 & 18.6 \\
\hline & $\begin{array}{l}\text { Esophagus V10Gy (\%) } \\
\text { (percentage volume of the esophagus irradiated more than or equal to } 10 \mathrm{~Gy} \text { ) }\end{array}$ & 53 & 39.4 \\
\hline & Esophagus V20Gy (\%) & 50.5 & 36.6 \\
\hline & Esophagus V30Gy (\%) & 49.4 & 34.8 \\
\hline & Esophagus V40Gy (\%) & 48.3 & 31.7 \\
\hline & Esophagus V50Gy (\%) & 31.6 & 28.8 \\
\hline & Esophagus V60Gy (\%) & 14.2 & 10.2 \\
\hline & Mean esophagus dose (Gy) & 27.6 & 21 \\
\hline \multirow[t]{18}{*}{ Patient 2} & Planning target volume (cc) & 326 & 76 \\
\hline & Lung V5Gy (\%) & 33.9 & 12.8 \\
\hline & Lung VS5Gy (cc) & 2457 & 3249 \\
\hline & Lung V10Gy (\%) & 19.3 & 8.8 \\
\hline & Lung V20Gy (\%) & 10.3 & 5.7 \\
\hline & Lung V30Gy (\%) & 5.8 & 2.7 \\
\hline & Lung V40Gy (\%) & 2.6 & 0.9 \\
\hline & Lung V50Gy (\%) & 1.4 & 0.1 \\
\hline & Lung V60Gy (\%) & 0.8 & 0 \\
\hline & Mean lung dose (Gy) & 7.3 & 3.5 \\
\hline & Mean dose of radiation pneumonitis area (Gy) & 18.9 & 17.7 \\
\hline & Esophagus V10Gy (\%) & 45.8 & 15.8 \\
\hline & Esophagus V20Gy (\%) & 42.3 & 12.1 \\
\hline & Esophagus V30Gy (\%) & 39.9 & 8.6 \\
\hline & Esophagus V40Gy (\%) & 30.9 & 5.2 \\
\hline & Esophagus V50Gy (\%) & 8.8 & 3.1 \\
\hline & Esophagus V60Gy (\%) & 3.4 & 1.5 \\
\hline & Mean esophagus dose (Gy) & 19.8 & 6.1 \\
\hline \multirow[t]{18}{*}{ Patient 3} & Planning target volume (cc) & 703 & 430 \\
\hline & Lung V5Gy (\%) & 39 & 27.2 \\
\hline & Lung VS5Gy (cc) & 2321 & 2776 \\
\hline & Lung V10Gy (\%) & 24.6 & 15.3 \\
\hline & Lung V20Gy (\%) & 14.9 & 9.4 \\
\hline & Lung V30Gy (\%) & 9.3 & 7.1 \\
\hline & Lung V40Gy (\%) & 5.6 & 4.9 \\
\hline & Lung V50Gy (\%) & 3 & 2.7 \\
\hline & Lung V60Gy (\%) & 0.4 & 0.3 \\
\hline & Mean lung dose (Gy) & 8.9 & 6.5 \\
\hline & Mean dose of radiation pneumonitis area $(\mathrm{Gy})$ & 34.6 & 30.7 \\
\hline & Esophagus V10Gy (\%) & 55.7 & 40.6 \\
\hline & Esophagus V20Gy (\%) & 45.8 & 29.7 \\
\hline & Esophagus V30Gy (\%) & 39.9 & 19.1 \\
\hline & Esophagus V40Gy (\%) & 32.4 & 13.5 \\
\hline & Esophagus V50Gy (\%) & 15.3 & 9.5 \\
\hline & Esophagus V60Gy (\%) & 7.3 & 5.5 \\
\hline & Mean esophagus dose (Gy) & 23.4 & 15 \\
\hline
\end{tabular}


Table IX. Continued

\begin{tabular}{|c|c|c|c|}
\hline & Dosimetric factors & $\begin{array}{l}\text { Actual radiation } \\
\text { therapy plan } \\
\text { with ENI }\end{array}$ & $\begin{array}{l}\text { Virtual radiation } \\
\text { therapy plan } \\
\text { without ENI }\end{array}$ \\
\hline \multirow[t]{18}{*}{ Patient 4} & Planning target volume (cc) & 423 & 186 \\
\hline & Lung V5Gy (\%) & 54.5 & 52.4 \\
\hline & Lung VS5Gy (cc) & 1089 & 1259 \\
\hline & Lung V10Gy (\%) & 28.9 & 26.8 \\
\hline & Lung V20Gy (\%) & 15.9 & 12 \\
\hline & Lung V30Gy (\%) & 9.3 & 7.1 \\
\hline & Lung V40Gy (\%) & 4 & 2.9 \\
\hline & Lung V50Gy (\%) & 0.5 & 0.3 \\
\hline & Lung V60Gy (\%) & 0 & 0 \\
\hline & Mean lung dose (Gy) & 9.9 & 8.7 \\
\hline & Mean dose of radiation pneumonitis area (Gy) & 31.2 & 29.8 \\
\hline & Esophagus V10Gy (\%) & 55.4 & 41.8 \\
\hline & Esophagus V20Gy (\%) & 53.1 & 26.5 \\
\hline & Esophagus V30Gy (\%) & 51.2 & 17.9 \\
\hline & Esophagus V40Gy (\%) & 48.4 & 9.9 \\
\hline & Esophagus V50Gy (\%) & 7 & 4.5 \\
\hline & Esophagus V60Gy (\%) & 0 & 0 \\
\hline & Mean esophagus dose (Gy) & 25.1 & 13 \\
\hline
\end{tabular}

All the four patients received volumetric modulated arc therapy.

inflammatory diseases caused mainly by smoking and radiation therapy, respectively. Using univariate analysis, Shi et al. reported that COPD was a significant factor $(p<0.05)$ in 11 patients with severe acute radiation pneumonitis among 94 NSCLC patients treated with IMRT (21). Using multivariate analysis, Inoue et al. reported that COPD was a significant factor $(p=0.002)$ in 44 patients with prolonged minimal radiation-induced pneumonitis after stereotactic body radiation therapy among 136 stage I lung cancer patients (22). Therefore, COPD might be a risk factor in relation to radiation pneumonitis. COPD should be treated along with lung cancer to minimize the influence of loss of normal lung function by chemoradiotherapy. Continuing medications such as an acting anti-muscarinic antagonist, acting beta agonist, or inhaled corticosteroid for COPD is important before and after chemoradiotherapy for NSCLC. Prohibiting smoking and vaccination against influenza and Streptococcus pneumoniae are also important (23).

\section{Conclusion}

ENI for NSCLC complicated with COPD decreased the rate of immunotherapy after definitive chemoradiotherapy. To increase the possibility of introducing immunotherapy, ENI should not be used for NSCLC complicated with COPD. Supportive care for NSCLC patients complicated with COPD should be done to minimize the loss of normal tissue function by chemoradiotherapy.

\section{Conflicts of Interest}

K Nishino had honoraria from Nippon Boehringer Ingelheim Co., Ltd., AstraZeneca K.K., Novartis Pharma K.K., Eli Lilly Japan K.K., Roche Diagnostics K.K., Chugai Pharma, and ONO PHARMACEUTICAL CO., LTD. K Nishino had research funding from Nippon Boehringer Ingelheim Co., Ltd. F Imamura had research funding from AstraZeneca. T Kumagai received a grant from Ono Pharmaceutical., MSD K.K., Chugai Pharmaceutical Co. Ltd., AstraZeneca K.K., Takeda Pharmaceutical Company Limited., Merck Serono Co., Ltd., Pfizer Japan Inc., Taiho Pharmaceutical Co.,Ltd., Nippon Boehringer Ingelheim Co., Ltd., Eli Lilly Japan K.K., Novartis Pharma K.K., and The Osaka Foundation for The Prevention of Cancer and Life-style related Diseases (Public Interest Incorporated Foundation). T Kumagai received personal fee from Ono Pharmaceutical., AstraZeneca K. K., Taiho Pharmaceutical Co. Ltd., MSD K.K., TEIJIN PHARMA LIMITED, Novartis Pharma K.K., Nippon Boehringer Ingelheim Co., Ltd., Eli Lilly Japan K.K., Pfizer Inc., Chugai Pharmaceutical Co. Ltd., Bristol-Myers Squibb K.K., and Takeda Pharmaceutical Company Limited.

\section{Authors' Contributions}

Conception and design: M Morimoto, and K Nishino. Acquisition of data: M Morimoto, K Nishino, K Wada, F Imamura, K Konishi, H Kuhara, M Tamiya, T Inoue, K Kunimasa, M Kimura, T Hirata, N Kanayama, M Toratani, H Kawachi, K Ohira, E Nakanishi, S Ohira, T Sagawa, M Miyazaki, T Kumagai, and T Teshima. Analysis and interpretation of data: M Morimoto, K Nishino, K Wada, F Imamura, K Konishi, H Kuhara, M Tamiya, T Inoue, K Kunimasa, M Kimura, T Hirata, N Kanayama, M Toratani, H Kawachi, K Ohira, E Nakanishi, 
S Ohira, T Sagawa, M Miyazaki, T Matsunaga (statistician), $\mathrm{T}$ Kumagai, and $\mathrm{T}$ Teshima. Writing, review, and/or revision of the manuscript: M Morimoto, K Nishino, K Wada, and K Kunimasa.

\section{Acknowledgements}

This study was supported by JSPS KAKENHI Grant Number 18K15616. The Authors deeply appreciate the investigator Toshiki Ikawa M.D. (Department of Radiation Oncology, Osaka International Cancer Institute, Osaka, Japan) who contributed to this study.

\section{References}

1 Antonia SJ, Villegas A, Daniel D, Vicente D, Murakami S, Hui R, Yokoi T, Chiappori A, Lee KH, de Wit M, Cho BC, Bourhaba M, Quantin X, Tokito T, Mekhail T, Planchard D, Kim YC, Karapetis CS, Hiret S, Ostoros G, Kubota K, Gray JE, Paz-Ares L, de Castro Carpeño J, Wadsworth C, Melillo G, Jiang H, Huang Y, Dennis PA and Özgüroğlu M: Durvalumab after chemoradiotherapy in stage III non-small-cell lung cancer. N Engl J Med 377: 1919-1929, 2017. PMID: 28885881. DOI: 10.1056/NEJMoa1709937

2 Antonia SJ, Villegas A, Daniel D, Vicente D, Murakami S, Hui $\mathrm{R}$, Kurata T, Chiappori A, Lee $\mathrm{KH}$, de Wit M, Cho BC, Bourhaba M, Quantin X, Tokito T, Mekhail T, Planchard D, Kim YC, Karapetis CS, Hiret S, Ostoros G, Kubota K, Gray JE, PazAres L, de Castro Carpeño J, Faivre-Finn C, Reck M, Vansteenkiste J, Spigel DR, Wadsworth C, Melillo G, Taboada M, Dennis PA and Özgüroğlu M: Overall survival with durvalumab after chemoradiotherapy in stage III NSCLC. N Engl J Med 379: 2342-2350, 2018. PMID: 30280658. DOI: 10.1056/NEJMoa1809697

3 Itazawa T, Tamaki Y, Komiyama T, Nishimura Y, Nakayama Y, Ito $\mathrm{H}$, Ohde Y, Kusumoto M, Sakai S, Suzuki K, Watanabe $\mathrm{H}$ and Asamura H: The Japan Lung Cancer Society-Japanese Society for Radiation Oncology consensus-based computed tomographic atlas for defining regional lymph node stations in radiotherapy for lung cancer. J Radiat Res 58: 86-105, 2017. PMID: 27609192. DOI: 10.1093/jrr/rrw076

4 The JLCS - JASTRO joint committee for developing an atlas of the regional lymph node stations in radiotherapy treatment planning for lung cancer, Komiyama T, Itazawa T, Tamaki Y, Nisimura Y, Nakayama Y, Ito H, Ohde Y, Kusumoto M, Sakai S, Suzuki K, Watanabe H and Asamura H: A consensus-based computed tomographic atlas for defining the regional lymph node stations in radiotherapy for lung cancer (in Japanese). Haigan 55: 189-205, 2015.

5 Imamura $\mathrm{F}$, Konishi $\mathrm{K}$, Uchida J, Nishino K, Okuyama T, Kumagai T, Kawaguchi $\mathrm{Y}$ and Nishiyama K: Novel chemoradiotherapy with concomitant boost thoracic radiation and concurrent cisplatin and vinorelbine for stage IIIA and IIIB non-small-cell lung cancer. Clin Lung Cancer 15: 281-286, 2014. PMID: 24656641. DOI: 10.1016/j.cllc.2014.02.001

6 Wada K, Kishi N, Kanayama N, Hirata T, Morimoto M, Konishi $\mathrm{K}$, Imamura $\mathrm{F}$, Teshima $\mathrm{T}$ and Ogawa $\mathrm{K}$ : Radiation dose escalation in accelerated hyperfractionated radiotherapy for stage III non-small-cell lung cancer. Anticancer Res 38: 5951-5958, 2018. PMID: 30275224. DOI: 10.21873/anticanres.12941

7 Wada K, Kishi N, Kanayama N, Hirata T, Ueda Y, Kawaguchi Y, Morimoto M, Konishi K, Imamura F, Ogawa K and Teshima
T: Predictors of acute radiation esophagitis in non-small cell lung cancer patients treated with accelerated hyperfractionated chemoradiotherapy. Anticancer Res 39: 491-497, 2019. PMID: 30591500. DOI: 10.21873 /anticanres.13139

8 Tsujino K, Hashimoto T, Shimada T, Yoden E, Fujii O, Ota Y, Satouchi M, Negoro S, Adachi S and Soejima T: Combined analysis of V20, VS5, pulmonary fibrosis score on baseline computed tomography, and patient age improves prediction of severe radiation pneumonitis after concurrent chemoradiotherapy for locally advanced non-small-cell lung cancer. J Thorac Oncol 9: 983-990, 2014. PMID: 24922010. DOI: 10.1097/JTO.00000 00000000187

9 De Ruysscher D, Faivre-Finn C, Moeller D, Nestle U, Hurkmans CW, Le Péchoux C, Belderbos J, Guckenberger M and Senan S: European Organization for Research and Treatment of Cancer (EORTC) recommendations for planning and delivery of high-dose, high precision radiotherapy for lung cancer. Radiother Oncol 124: 1-10, 2017. PMID: 28666551. DOI: 10.1016/j.radonc.2017.06.003

10 Belderbos JS, Kepka L, Spring Kong FM, Martel MK, Videtic GM and Jeremic B: Report from the International Atomic Energy Agency (IAEA) consultants' meeting on elective nodal irradiation in lung cancer: non-small-cell lung cancer (NSCLC). Int J Radiat Oncol Biol Phys 72: 335-342, 2008. PMID: 18793953. DOI: 10.1016/j.ijrobp.2008.04.081

11 Bradley JD, Paulus R, Komaki R, Masters G, Blumenschein G, Schild S, Bogart J, Hu C, Forster K, Magliocco A, Kavadi V, Garces YI, Narayan S, Iyengar P, Robinson C, Wynn RB, Koprowski C, Meng J, Beitler J, Gaur R, Curran W Jr. and Choy $\mathrm{H}$ : Standard-dose versus high-dose conformal radiotherapy with concurrent and consolidation carboplatin plus paclitaxel with or without cetuximab for patients with stage IIIA or IIIB non-smallcell lung cancer (RTOG 0617): a randomised, two-by-two factorial phase 3 study. Lancet Oncol 16: 187-199, 2015. PMID: 25601342. DOI: 10.1016/S1470-2045(14)71207-0

12 Japanese Society for Radiation Oncology: Radiation therapy planning guideline 2016 edition. Tokyo: Kanahara Co., Ltd., 2016.

13 Perez CA, Stanley K, Grundy G, Hanson W, Rubin P, Kramer S, Brady LW, Marks JE, Perez-Tamayo R, Brown GS, Concannon JP and Rotman M: Impact of irradiation technique and tumor extent in tumor control and survival of patients with unresectable non-oat cell carcinoma of the lung: report by the Radiation Therapy Oncology Group. Cancer 50: 1091-1099, 1982. PMID: 6286087. DOI: 10.1002/1097-0142(19820915)50:6<1091::aidcncr2820500612>3.0.co;2-0

14 Yuan S, Sun X, Li M, Yu J, Ren R, Yu Y, Li J, Liu X, Wang R, Li B, Kong L and Yin Y: A randomized study of involved-field irradiation versus elective nodal irradiation in combination with concurrent chemotherapy for inoperable stage III nonsmall cell lung cancer. Am J Clin Oncol 30: 239-244, 2007. PMID: 17551299. DOI: 10.1097/01.coc.0000256691.27796.24

15 Topkan E, Guler OC and Yildirim BA: Omission of elective nodal irradiation has no impact on isolated elective nodal failure and survival outcomes in stage III non-small-cell lung cancer patients treated with definitive concurrent chemoradiotherapy. Ann Oncol 26: i24, 2015. DOI: https://doi.org/10.1093/annonc/mdv049.08

16 Li R, Yu L, Lin S, Wang L, Dong X, Yu L, Li W and Li B: Involved field radiotherapy (IFRT) versus elective nodal irradiation (ENI) for locally advanced non-small cell lung cancer: a meta-analysis of incidence of elective nodal failure 
(ENF). Radiat Oncol 11: 124, 2016. PMID: 27655339. DOI 10.1186/s13014-016-0698-3

17 Schild SE, Pang HH, Fan W, Stinchcombe TE, Vokes EE, Ramalingam SS, Bradley JD, Kelly K and Wang X: Exploring radiotherapy targeting strategy and dose: A pooled analysis of cooperative group trials of combined modality therapy for stage III NSCLC. J Thorac Oncol 13: 1171-1182, 2018. PMID: 29689435. DOI: $10.1016 /$ j.jtho.2018.04.011

18 De Ruysscher D, Faivre-Finn C, Nackaerts K, Jordan K, Arends J, Douillard JY, Ricardi U and Peters S: Recommendation for supportive care in patients receiving concurrent chemotherapy and radiotherapy for lung cancer. Ann Oncol 31: 41-49, 2020. PMID: 31912794. DOI: 10.1016/j.annonc.2019.10.003

19 Abal Arca J, Parente Lamelas I, Almazán Ortega R, Blanco Pérez J, Toubes Navarro ME and Marcos Velázquez P: Cáncer de pulmón y EPOC: una asociación frecuente [Lung cancer and COPD: a common combination] (in Spanish, abstract). Arch Bronconeumol 45: 502-507, 2009. PMID: 19748721. DOI: 10.1016/j.arbres.2009.07.005

20 Loganathan RS, Stover DE, Shi W and Venkatraman E: Prevalence of COPD in women compared to men around the time of diagnosis of primary lung cancer. Chest 129: 1305-1312, 2006. PMID: 16685023. DOI: 10.1378/chest.129.5.1305
21 Shi A, Zhu G, Wu H, Yu R, Li F and Xu B: Analysis of clinical and dosimetric factors associated with severe acute radiation pneumonitis in patients with locally advanced non-small cell lung cancer treated with concurrent chemotherapy and intensitymodulated radiotherapy. Radiat Oncol 5: 35, 2010. PMID: 20462424. DOI: $10.1186 / 1748-717 X-5-35$

22 Inoue T, Shiomi H and Oh RJ. Stereotactic body radiotherapy for Stage I lung cancer with chronic obstructive pulmonary disease: special reference to survival and radiation-induced pneumonitis. J Radiat Res 56: 727-734, 2015. PMID: 25887042. DOI: $10.1093 / \mathrm{jrr} / \mathrm{rrv019}$

23 The Japanese Respiratory Society: The JRS Guidelines for the Management of Chronic Obstructive Pulmonary Disease 5th edition. Tokyo: Medical Review Co., Ltd., 2018.
Received October 3, 2020

Revised October 8, 2020

Accepted October 9, 2020 